HoST - Journal of History of Science and Technology

Vol. 13, no. 2, December 2019, pp. 1-4

10.2478/host-2019-0010

\title{
Introduction: Taking animals seriously in studies of science and technology
}

\author{
Richie Nimmo \\ University of Manchester
}

Richie.Nimmo@Manchester.ac.uk

In the multiple overlapping fields that make up the history of science and technology it is widely understood that society, nature, science, and technology, are so thoroughly entangled that conceiving of any one of these as a discrete domain is untenable. Histories of science and technology are thus histories of mediations, inscriptions, enactments, and materialisations of social and political relations, and scientific and technological objects and practices are assemblages of "social" and "natural" or human and nonhuman entities, forces and actants. What is still often backgrounded, however, in the relational ontologies that inform studies of these human-nonhuman entanglements, is what we might provisionally refer to as nonhuman nonhumans, that is to say, living nonhumans such as nonhuman animals, as distinct from the human nonhumans of technologies and scientific objects. Whereas the latter can still conceivably be thought of as essentially human constructs, ultimately deriving their agency and meaning from human social actors, this is far more problematic with reference to living nonhumans such as nonhuman animals. In this sense mainstream histories of science and technology are

(C) 2019 Richie Nimmo. This is an open access article licensed under the Creative Commons Attribution-NonCommercial-NoDerivs License (http://creativecommons.org/licenses/by-nc-nd/3.0/). 
still by and large residually human-centred, in their backgrounding or exclusion of nonhuman animals. This is problematic, given the often-pivotal role of animals in scientific "discoveries," controversies and developments. Insofar as animals have entered into histories of science and technology, they have often appeared either as passive objects of scientific knowledge and technological manipulation, or as hollowed out relational nodes in heterogeneous networks rendered theoretically equivalent to technological nonhumans by being shorn of their vital creatureliness, their flesh and blood animality. Taking nonhuman animals seriously means undoing this by tracing how, in their very liveliness and irreducibility, animals shape scientific and socio-technical knowledge-practices and often trouble not only objectivist scientific epistemologies but also anthropocentric visions of the science-technology-society nexus by opening up zones of productive uncertainty and indeterminacy.

Rising to this challenge, this themed issue brings together an exciting collection of leading and emerging scholars from a variety of disciplines and fields, encompassing the history of science, technology and medicine, sociological human-animal studies, and science, technology and society. Each is critically engaged in exploring in different yet complementary empirical and theoretical registers the unsettling multispecies complexities and more-than-human histories of science and technology.

In her article Amanda Rees examines the development of the socio-ecology of marine mammals by tracing the entangled emergence, propagation and cross-fertilisation of the socio-technical practices which enabled these animals to be studied. She notes that the study of animals as they lived in their natural habitats rather than in laboratory conditions emerged from the late 1950s, initially with reference to terrestrial and avian animals, with marine mammals excluded by virtue of the difficulties presented to humans by their aquatic environments. Yet by the twentyfirst century wild-living marine mammals including whales, dolphins and seals were subject to multi-generational studies in which individuals and their complex social interrelationships were being tracked in depth. Rees unpacks how this transition was accomplished in all its minute and heterogeneous ingredients, and traces how techniques and practices were adapted from those of other groups, including diverse lay practitioners from sailors to zookeepers, to make it possible. She also unearths how these efforts were entangled with those involved in displaying marine mammals on land, and explores the ethical as well as pragmatic issues arising from this intersection. Throughout Rees' analysis the agency of the animals in shaping the emergence of cetology is foregrounded, underlining their active contribution as subjects to the development of those knowledge-practices.

Robert Kirk's article considers the pivotal 1980s controversy around the treatment and fate of the monkeys involved in Edward Taub's experimental neuroscience research at the Institute of Behavioral Research in Silver Spring, Maryland. Kirk traces in detail how the episode brought into conflict different institutions, authorities and social actors, from Taub himself to the 
National Institutes of Health (NIH), to ethologists and veterinarians, to the emergent People for the Ethical Treatment of Animals (PETA). His analysis challenges the conventional reading of this controversy as an example of the conflict between anthropocentric human interests and animal rights values, elaborating instead a more socio-historically situated and less polarised reading in which it was also a pivotal encounter between distinct cultures of laboratory animal care with their own embedded knowledges, norms and assumptions, giving rise to a generative moment from which new approaches to animal care and welfare emerged.

In her article Maisie Tomlinson looks at the hitherto under-examined methodology of animal behavioural expertise known as Qualitative Behavioural Analysis (QBA). Developed principally by Francois Wemelsfelder, QBA seeks to incorporate interpretative assessments of the expressive qualities of animal behaviour and animal emotions into a scientific methodology for the assessment of animal welfare. Whilst now widely recognised, it remains contentious for some due to its perceived "subjective" or "anthropomorphic" approach, which rejects the sceptical reduction of animals to objects of knowledge made up of atomised parts, which can be neutrally recorded by a suitably detached observer. Tomlinson's article, grounded in empirical ethnographic research on QBA as science in action, unfolds the "whole animal" relational philosophy underpinning this innovative methodology, and examines its significance and implications by unpacking the socio-historically inscribed politics of knowledge that shape its enactment in practice as well as ongoing debates around its validity.

Sainath Suryanarayanan moves from the focus on scientific studies of land and marine mammals to sciences of social insects, closely examining the epistemological and ontological underpinnings of the emerging field of sociogenomics in relation to the changing use of social insects as models of social behaviour. Pivotal for sociogenomics is "the genome," where social and environmental "information" is held to interact with genetic variation in ways that influence social behavior through shifts in gene expression. Suryanarayanan shows how the advent of genome sequencing technologies, comparative genomics, and informational tools for identifying patterns of association across diverse datasets, has facilitated the enrollment of social insects such as ants, bees and wasps in the project of identifying genetic networks ostensibly underlying autism, novelty-seeking and aggression, with evolutionary links made to humans. His article draws widely on the literature of social insect biology as well as historians and philosophers of science to explore how the historical development of social insect research has shaped approaches in contemporary sociogenomics, especially in terms of changing notions of sociality and individuality.

My own article moves from the previous principal focus on scientific epistemologies and knowledge-practices towards the ontologies and materialities of technology and the animaltechnology interface. In a theoretically-driven analysis, I critically assess the contribution of Foucauldian poststructuralist approaches to the human-animal technology nexus in industrial 
animal agriculture, situated in light of key recent literature from human-animal studies addressing animals and biopolitics. Drawing upon archive sources, these theoretical debates are worked through the historical case of the emergence and development of the earliest mechanical devices for milking cows, tracing the development of milking machines as a remarkably messy, ad-hoc and iterative socio-technical process, in which the designers were often thwarted by the corporeality of the animals and forced to rethink their approach in their attempts to negotiate the difficult interface between living animal and machine.

\section{Acknowledgements}

I am grateful to the HoST journal editors, as well as the contributing authors, for their diligence and patience throughout the editorial process. 